\title{
IF ALL NORMAL MOORE SPACES ARE METRIZABLE, THEN THERE IS AN INNER MODEL WITH A MEASURABLE CARDINAL
}

\author{
BY \\ WILLIAM G. FLEISSNER
}

\begin{abstract}
We formulate an axiom, HYP, and from it construct a normal, metacompact, nonmetrizable Moore space. HYP unifies two better known axioms. The Continuum Hypothesis implies HYP; the nonexistence of an inner model with a measurable cardinal implies HYP. As a consequence, it is impossible to replace Nyikos' "provisional" solution to the normal Moore space problem with a solution not involving large cardinals. Finally, we discuss how this space continues a process of lowering the character for normal, not collectionwise normal spaces.
\end{abstract}

1. The normal Moore space problem. In 1931 F. B. Jones [J] asked whether all normal Moore spaces were metrizable. Towards this end, he proved that assuming $2^{\aleph_{0}}<2^{\aleph_{1}}$, separable normal Moore spaces are metrizable. R. H. Bing [B] showed that a Moore space is metrizable if and only if it is collectionwise normal (i.e. every discrete collection of closed sets can be simultaneously separated by disjoint open sets). If there is a $Q$-set, a special subset of the real line, then Example E of Bing's paper is a separable, normal, nonmetrizable Moore space. Soon after the formulation of Martin's Axiom, it was shown that $\mathrm{MA}_{\kappa_{1}}$ implied the existence of $Q$-sets, hence also of separable, normal, nonmetrizable Moore spaces [ $\left.\mathbf{T}_{1}\right]$.

In 1974 , the author $\left[\mathbf{F}_{1}\right]$ showed that assuming Gödel's axiom of constructibility normal spaces of character $\leqslant 2^{\aleph_{0}}$ are collectionwise Hausdorff. One consequence of this result is that if there is a construction of a normal, nonmetrizable (equivalently, not collectionwise normal) Moore space with ZFC, the usual axioms of set theory, then the closed sets that cannot be separated are not points-in fact, not even Lindelöf.

Above, we saw that it is consistent with ZFC that normal nonmetrizable Moore spaces exist. P. J. Nyikos [N] gave a "provisional" solution to the normal Moore space conjecture by showing that the Product Measure Extension Axioms (PMEA) implies that normal Moore spaces are metrizable. Nyikos called this a "provisional" solution because a large cardinal (specifically, a strongly compact cardinal) was used in the construction of the model of ZFC + PMEA. Nyikos' result posed the question of whether a large cardinal assumption is necessary (rather than just convenient) for

Received by the editors August 25, 1981.

1980 Mathematics Subject Classification. Primary 03E35, 54E30.

Key words and phrases. Normal Moore space, full sets, large cardinal consistency results.

${ }^{1}$ Supported by NSF Grant MCS-79-01848. 
the metrization theorem. See $\left[\mathbf{T}_{2}\right]$ for a fuller history of the normal Moore space problem.

In the summer of 1980 , the author constructed a normal nonmetrizable Moore space, not from a new axiom, but from the familiar Continuum Hypothesis $(\mathrm{CH})$. Later that year, he showed that the construction of the space can be done from a general axiom of which $\mathrm{CH}$ is the simplest case. The importance of the other cases is that they complete the solution of the normal Moore space problem. If the other cases fail, then work of Jensen [DJ, JD] shows that there is an inner model with a measurable cardinal. Hence, Nyikos' "provisional" solution cannot be improved by deleting the large cardinal hypothesis.

2. Terminology and notation. Our topological terminology conforms with current American usage, and can be found in [E, pp. 411-414]. We will not need to define Moore space; we will use instead the notion of uniform base which was defined by Aleksandrov and shown equivalent to metacompact Moore space by Arhangel'skij [E]. A family, $\mathcal{G}$, of open sets is called a uniform base if for every infinite subset, $\mathscr{U}$, of $\mathcal{G}$, if $x \in \cap \mathscr{U}$ then $\mathcal{U}$ is a neighborhood base at $x$.

Our set theoretic notation is standard and can be found in [Je] or [K]. In particular, $f \mid A$ denotes the function $f$ restricted to the set $A$; an ordinal is the set of smaller ordinals; $\omega$ is the first infinite ordinal; and $\omega_{1}$ is the first uncountable ordinal. We reserve $\alpha, \beta, \gamma, \delta$ for ordinals, $i, j, k, l, m, n$ for natural numbers, and $\kappa, \lambda, \mu$ for infinite cardinals. We always use $\kappa$ for the cardinal satisfying HYP (defined in $\S 3$ ); $\kappa^{+}$is the cardinal successor of $\kappa$. We definitely include the possibility $\kappa=\omega, \kappa^{+}=\omega_{1}$. We reserve $\rho, \sigma, \tau, \nu$ for finite functions whose domains are natural numbers (i.e. the formalization of "finite sequence") and range contained in the set $E$ of HYP; $f, g$ are functions with domain $\omega$ (i.e. the formalization of infinite sequence) and range contained in the set $E$ of HYP.

3. Axiom HYP and large cardinals. We begin by presenting in detail the axiom which we use to construct the space. Let HYP be the axiom which assets "There exist $\kappa,\left(\kappa_{n}\right)_{n \in \omega}$, and $E$ satisfying

$$
\left(\kappa_{n}\right)_{n \in \omega} \text { is an increasing sequence of cardinals cofinal in } \kappa
$$

$$
\begin{gathered}
\text { for all } n \in \omega, 2^{\kappa_{n}}<\kappa, \\
2^{\kappa}=\kappa^{+}, \\
E \subset\left\{\delta \in \kappa^{+}: c f(\delta)=\omega\right\} \text { and } E \text { is stationary in } \kappa^{+}, \\
\text {for all } \beta<\kappa^{+}, E \cap \beta \text { is not stationary in } \beta^{\prime \prime} .
\end{gathered}
$$

In informal language, (1) says that $\kappa$ is a strong limit cardinal of cofinality $\omega$, (2) says that the $\kappa$-continuum hypothesis holds, and (3) says that $E$ is a nonreflecting stationary subset of $\omega$ limits less than $\kappa^{+}$.

Throughout this paper, let $\kappa,\left(\kappa_{n}\right)_{n \in \omega}$, and $E$ satisfy HYP ((1)-(3) above). Further, we fix for each $\delta \in E$ an increasing sequence, $\left(\delta_{i}\right)_{i \in \omega}$, of nonlimit ordinals cofinal in $\delta$. We will not use (3b) directly, but rather the following consequence.

LEMMA 1. For all $\beta<\kappa^{+}$, there is a function, $m_{\beta}$, from $E \cap \beta$ to $\omega$ such that for each pair $\delta, \eta$ of distinct elements of $E \cap \beta$, if $m=\max \left\{m_{\beta}(\delta), m_{\beta}(\eta)\right\}$ then $\delta_{m} \neq \eta_{m}$. 
Proof. By induction on $\beta$. (In the case $\kappa=\omega$, there is a straightforward, noninductive proof, which is left to the reader.) If $\beta=0$, let $m_{\beta}=\varnothing$; if $\beta=\alpha+2$, let $m_{\beta}=m_{\alpha+1}$. So assume $\beta=\alpha$ or $\beta=\alpha+1$ where $\alpha$ is a limit ordinal. By (3b) there is $C$, club in $\alpha$ and disjoint from $E$. In the case where $\beta=\alpha+1$ and $\alpha \in E$, we can assume $\left\{\alpha_{i}: i \in \omega\right\} \subset C$. Fix $\delta \in E \cap \alpha$; let $\gamma=\sup (C \cap \delta)$ and $\zeta=$ $\inf (C-\delta)$. Choose $i$ so that $\delta_{i}>\gamma$ and $m_{\zeta}(\delta) \geqslant i$. Set $m_{\alpha}(\delta)=i$. If $\beta=\alpha+1$ and $\alpha \notin E$, set $m_{\beta}=m_{\alpha}$. If $\beta=\alpha+1$ and $\alpha \in E$, set $m_{\beta}=m_{\alpha} \cup\{\langle\alpha, 0\rangle\}$.

The existence of a normal, nonmetrizable Moore space follows from several extra axioms of set theory. The first known was Martin's Axiom MA $\boldsymbol{\aleph}_{1}$. Subsequently, Martin's Axiom was rephrased to include $\mathrm{CH}$ as a special case. $\mathrm{CH}$ is also a special case of HYP. To see this, set $\kappa=\omega, \kappa_{n}=n$, and $E=\left\{\delta \in \omega_{1}: \delta\right.$ is a limit ordinal $\}$. Then (1) and (3) are automatically satisfied, and (2) is the ordinary Continuum Hypothesis. We urge readers to consider only this case on first reading. After this specific case is understood, it is straightforward to check that only properties (1), (2), (3) were used in the proof.

While the case $\kappa=\omega$ is the simplest case, there are other cardinals $\kappa$ which could satisfy HYP. To illustrate, we define inductively the Beth numbers. Set $\beth_{0}=\omega$; $\beth_{\alpha+1}=2^{Z_{\alpha}}$; and for limit ordinals $\gamma, \beth_{\gamma}=\sup \left\{\beth_{\alpha}: \alpha<\gamma\right\}$. Every cardinal of the form $\beth_{\delta}$, where $c f(\delta)=\omega$ satisfies (1).

The only known proof of the consistency of ZFC + NMSC (the assertion that all normal Moore spaces are metrizable) is to start with a model with a strongly compact cardinal, $\kappa$, and then add $\kappa$ random reals. In the resulting model, PMEA holds, and PMEA implies NMSC. It is natural to ask whether a large cardinal is necessary to get a model of NMSC. The importance of the case HYP holding for $\kappa>\omega$ is to show that a large cardinal is necessary. We do not construct the inner model with a measurable cardinal in this paper, but simply use results of Jensen, Dodd, and Mitchell. The basic situation is illustrated in Figure 1.

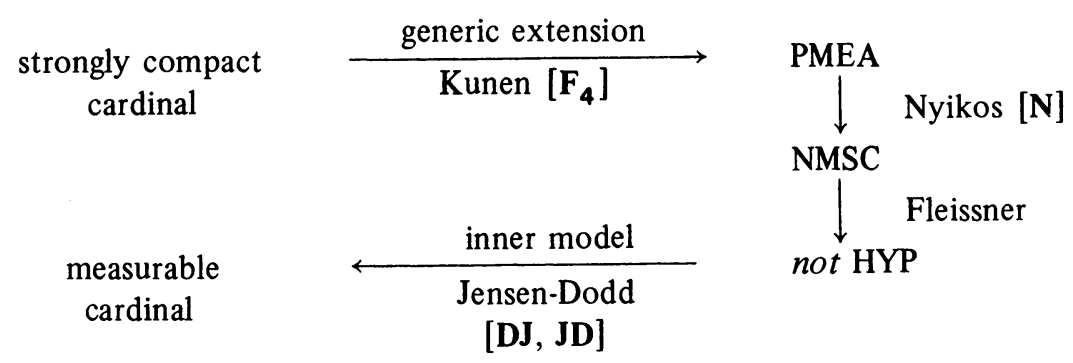

\section{Figure 1}

In the figure the downward arrows represent implications, while the horizontal arrows represent constructions of one model of set theory from another. Thus they represent that the consistency of one theory implies the consistency of another theory. Mitchell has constructed from not HYP inner models with large cardinals stronger than measurable [Mt]. 
NMSC fails if there is just one $\kappa$ satisfying (1), (2), and (3). The construction of an inner model with a large cardinal requires just one $\kappa>\omega$ where (1) holds but either (2) or (3) fails. The failure of (2) or (3) at every of the proper class of $\kappa$ 's satisfying (1) does not seem to yield significantly stronger consistency results than the failure at just one such $\kappa$. In particular, Mitchell's results give much stronger consistency than the consistency of a proper class of measurable cardinals.

Let us warn against trying to prove a result of the form "If there is a normal, nonmetrizable Moore space then there is one of cardinality $\lambda$ " where $\lambda$ is, say, $\beth_{\omega}^{+}$. Start with a model $M_{0} \vDash$ " $\kappa$ is strongly compact." By Solovay, $M_{0}$ ₹ "if $\mu>\kappa$ satisfies (1), then $\mu$ satisfies (2)." Now add $\kappa$ random reals to get $M_{1} \vDash$ PMEA. Choose a cardinal $\mu$ which satisfies (1) and is greater than $\lambda$ of the conjecture. It is well known that we can add a subset $E$ of $\mu^{+}$satisfying (3) without adding a set of ordinals of cardinality $<\mu$, to get a model $M_{2}$ ₹ " no normal nonmetrizable Moore space of cardinality $\lambda$, and there is a normal nonmetrizable Moore space of cardinality $\mu^{+}$."

4. Visualizing the space. In this section, we describe Heath's $V$-space, $H$, and explain how the space of this paper (not yet defined) "looks like" the $V$-space. This section is not strictly necessary. However, some readers may want a geometric picture to go with the abstract definition of the space.

The point set of $H$ consists of the $x$-axis and the upper half-plane. Points of the upper half-plane are isolated. The $n$th basic open neighborhood, $B_{n}(P)$, of a point, $P$, on the $x$-axis consists of the two line segments from $P$ to the line $y=\frac{1}{n}$ with slopes of +1 and -1 (i.e. a point with two arms-a " $V$ "). It is routine to verify that we have defined a space with a uniform base.

$H$ is not normal because we cannot separate $Q=\{(x, 0): x$ is rational $\}$ and $I=\{(x, 0): x$ is irrational $\}$. Suppose that $U$ is an open set containing $I$. For $n \in \omega$, set $A_{n}=\left\{x \in R: B_{n}(x, 0) \subset U\right\}$. By the Baire Category Theorem, there is an interval $(a, b)$ and a $n \in \omega$ such that $A_{n}$ is dense in $(a, b)$. If $x$ is a rational in $(a, b)$, then $(x, 0) \in \bar{U}$. Hence $H$ is not normal.

The space we will define below will "look like" $H$. The set $F$ will correspond to the $x$-axis. The set of pairs, $(\rho, \tau)$, of functions from $n$ to $\omega_{1}$ will correspond to the line $y=\frac{1}{n}$. We place "cubes" of functions indexed by $(\rho, \tau)$ along the line $y=\frac{1}{n}$. A basic open set, indexed by $\sigma$, consists of a piece of $F$, together with two "arms". One arm contains corners of some cubes indexed by $(\rho, \tau)$ where $\sigma \subseteq \rho$; the other arm contains corners of some cubes where $\sigma \subseteq \tau$.

Corresponding to the use of the Baire Category Theorem is the lemma on stafull sets. Roughly, it says that when $F$ is covered by basic sets, for some $n \in \omega$, there are so many $n$th basic open sets that some have to be entwined, and hence intersect.

5. Construction of the space. Let $F$ be the set of functions from $\omega$ to $E$. For $n \in \omega$, set $\Sigma_{n}=\{f \mid n: f \in F\}$ and set $\Sigma=\cup_{n \in \omega} \Sigma_{n}$. For $\sigma \in \Sigma$, set $[\sigma]=\{f \in F: \sigma \subset f\}$. Then $\{[\sigma]: \sigma \in \Sigma\}$ is a basis for a completely metrizable, strongly zero-dimensional topology on $F$.

Let $Z$ be the family of subsets, $Z$, of $\Sigma$ satisfying card $Z \leqslant \kappa$ and for some $n \in \omega$, $Z \subset \Sigma_{n}$. By (2), we can list $\mathscr{Z}$ as $\left\{Z_{\alpha}: \alpha<\kappa^{+}\right\}$. For $\sigma \in \Sigma$, define $\sigma^{*}$ to be the 
greatest ordinal in range $\sigma$. For $\beta<\kappa^{+}$set $\Sigma^{\beta}=\left\{\sigma: \sigma^{*}<\beta\right\}$ and $\mathscr{Z}(\beta)=\left\{Z_{\alpha}\right.$ : $\alpha<\beta\}$. By induction on $n \in \omega$, we define, for $\sigma \in \Sigma_{n}$ and $m \in \omega$, the sets $A(\sigma, m)$ to satisfy

$$
\begin{gathered}
\operatorname{card} A(\sigma, m)=\kappa_{m} \quad\left(\operatorname{card} A(\sigma, m) \leqslant m^{2} \text { if } \kappa=\omega\right), \\
\bigcup_{m \in \omega} A(\sigma, m)=\mathscr{Z}\left(\sigma^{*}\right) \cap\left(\bigcup_{k<n} \Sigma_{k}\right),
\end{gathered}
$$

$$
\text { if } \sigma \subset \sigma^{\prime} \text { and } m \leqslant m^{\prime} \text {, then } A(\sigma, m) \subset A\left(\sigma^{\prime}, m^{\prime}\right) \text {. }
$$

Let $Q_{k}$ be the set of triples $\langle g, \rho, \tau\rangle$ which satisfy

$$
\begin{gathered}
\rho, \tau \in \Sigma_{k}, \\
\text { for all } i<k-1, \quad \rho(i)<\tau(i)<\rho(i+1)<\tau(i+1), \\
g \text { is a function from } \mathscr{Z}\left(\rho^{*}\right) \text { to }\{0,1\} .
\end{gathered}
$$

Set $Q=\cup_{k \in \omega} Q_{k}$. For $\sigma \in \Sigma_{n}$, define $G(\sigma)$ to be the set of triples $\langle g, \rho, \tau\rangle \in Q$ satisfying (we will use $\Sigma^{\rho \mid m}$ for $\Sigma^{(\rho \mid m)^{*}}$ )

$$
\text { either } \sigma \subset \rho \text { or } \sigma \subset \tau \text {, }
$$$$
\text { for all } i<n, \quad \rho(0)_{i}=\tau(0)_{i},
$$

Set $B(\sigma)=[\sigma] \cup G(\sigma)$.

The point set of our space, $X$, is $F \cup Q$. A basis for the topology on $X$ is $\{B(\sigma)$ : $\sigma \in \Sigma\} \cup\{\{q\}: q \in Q\}$. That we have indeed defined a basis follows from (6). It is easy to see that $X$ is $T_{1}$. If $\{[\sigma \mid 1]\} \in A(\sigma, n)$, where $\sigma \in \Sigma_{n}$, then $B(\sigma)$ is both open and closed (use (11), (12), and (8)); hence $X$ is regular. Finally, set

$$
\mathcal{G}_{n}=\left\{B(\sigma): \sigma \in \Sigma_{n}\right\} \cup\left\{\{q\}: q \in Q_{k} \text { where } k<n\right\} .
$$

For every infinite subset $\mathscr{Q}$ of $\cup_{n \in \omega} \mathcal{G}_{n}$, if $x \in \cap \mathscr{U}$, then $\mathscr{Q}$ is a basis at $x$. Hence $X$ is a metacompact Moore space.

6. $X$ is normal. Assume that $H$ and $K$ are disjoint closed subsets of $X$. First, we see that we need to consider only the case where $H \cup K \subseteq F$. For if $H \cap F \subset U$ and $K \cap F \subset V$, where $U$ and $V$ are disjoint open subsets of $X$, then $U \cup(H \cap Q)-K$ and $V \cup(K \cap Q)-H$ are disjoint, open, and contain $H$ and $K$, respectively. Second, define, for $n \in \omega, H_{n}=\bigcup\left\{[\sigma]:[\sigma] \cap K=\varnothing\right.$ and $\left.\sigma \in \Sigma_{n}\right\}$ and define $K_{n}$ similarly. By the "regular plus Lindelöf implies normal" argument, if we can separate, for each $n \in \omega$, both pairs $H_{n}$ and $F-H_{n}, K_{n}$ and $F-K_{n}$, then we can separate $H$ and $K$. We summarize these reductions in

LEMMA 2. To show that $X$ is normal, it suffices to separate, for each $n \in \omega$ and each $Z \subset \Sigma_{n}$, the set $H_{z}=\cup\{[\sigma]: \sigma \in Z\}$ and $K_{Z}=F-H_{Z}$.

Now let $n, Z, H_{Z}$, and $K_{Z}$ be as in Lemma 2. Define

$$
C=\left\{\gamma \in \kappa^{+}: \text {if } \beta<\gamma, \text { then } Z \cap \Sigma^{\beta}=Z_{\alpha} \text { for some } \alpha<\gamma\right\} \text {. }
$$


$C$ is closed and unbounded in $\kappa^{+}$. For $\beta \in \kappa^{+}$, define $\gamma(\beta)$ to be the least element of $C$ greater than $\beta$. For $\sigma \in \Sigma_{n+3}$, define $j(\sigma) \geqslant n+3$ to satisfy

$$
\text { if } \begin{gathered}
\gamma(\sigma(n))<\sigma(n+2) \text {, then } Z \cap \Sigma^{\sigma(n)} \in A(\sigma, j(\sigma)) . \\
j(\sigma) \geqslant m_{\gamma(\sigma(n+1))}(\sigma(0)) .
\end{gathered}
$$

Set $W_{\sigma}=\cup\left\{B(\rho): \sigma \subseteq \rho \in \Sigma_{j(\sigma)}\right\}$. To separate $H_{Z}$ and $K_{Z}$, it will suffice to show that if $\sigma, \nu \in \Sigma_{n+3}, \sigma \mid n \in Z$, and $\nu \mid n \notin Z$, then $W(\sigma) \cap W(\nu)=\varnothing$. Aiming for a contradiction, assume that $\langle g, \rho, \tau\rangle \in W(\sigma) \cap W(\nu)$. By (10) either $\sigma \subseteq \rho$ or $\sigma \subseteq \tau$. Without loss of generality, assume $\sigma \subseteq \rho$; then also $\nu \subseteq \tau$.

Case 1. $\gamma(\nu(n))<\sigma(n+2)$. Then $Z \cap \Sigma^{\sigma(n)} \in A(\sigma, j(\sigma))$ and $Z \cap \Sigma^{\nu(n)} \in$ $A(\nu, j(\nu))$. Since $\sigma \mid n \in Z$ and $\nu \mid n \notin Z$, we have by (12)

$$
1=g\left(Z \cap \Sigma^{\rho(n)}\right)=0 .
$$

Case 2. $\sigma(n+2) \leqslant \gamma(\nu(n))$. Then $\gamma(\sigma(n+1))=\gamma(\nu(n+1))$. By $(8), \sigma(0) \neq \nu(0)$, so from (16) and Lemma $1, m=\max \{j(\sigma), j(\nu)\}$ satisfies $\rho(0)_{m} \neq \tau(0)_{m}$. Contradiction to (11).

7. $X$ is not metrizable. The following notion breaks the demonstration into several short combinatorial arguments.

Definition. A subset, $S$, of $\Sigma_{n}$ is stafull if for all $\sigma \in S$ and $j<n,\{\tau(j)$ : $\sigma \mid j \subset \tau \in S\}$ is stationary.

Lemma 3. (a) If $\cup\{[\sigma]: \sigma \in T\}=F$, then for some $n, T \cap \Sigma_{n}$ has a stafull subset.

(b) Suppose that $S \subset \Sigma_{n}$ is stafull, and that $h: S \rightarrow \kappa^{+}$satisfies for all $\sigma \in S$, $h(\sigma)<\sigma(0)$. Then there is a stafull $S^{\prime} \subset S$ such that $h \mid S^{\prime}$ is constant.

(c) If $S^{\prime} \subset \Sigma_{n}$ is stafull, then for each $\beta \in \kappa^{+}$, there is $W=\left\{\sigma_{\alpha}: \alpha<\beta\right\} \subset S^{\prime}$ such that

$$
\sigma_{\alpha}(i)<\sigma_{\alpha^{\prime}}\left(i^{\prime}\right) \quad \text { iff } i<i^{\prime} \text { or } i=i^{\prime} \text { and } \alpha<\alpha^{\prime} \text {. }
$$

Proof. (a) Assume that for all $n \in \omega, T \cap \Sigma_{n}$ does not have a stafull subset. We construct $f \notin \cup\{[\sigma]: \sigma \in T\}$. By induction on $i \in \omega$ define $f \mid i$ so that for each $n>i$ and $T^{\prime} \subset T \cap \Sigma_{n}$ there is $\tau \in T^{\prime}$ such that either $f \mid i \not \subset \tau$ or there is $j$, $i \leqslant j<n$, such that $\left\{\rho(j): \tau \mid j \subset \rho \in T^{\prime}\right\}$ is not stationary.

(b) By induction on $i$ define a function $h_{i}$ so that $h_{0}=h$, domain $h_{1}=\{\sigma \mid n-i$ : $\sigma \in S\}$ and for $i>0$, if $\rho \in$ domain $h_{i}$, then $\left\{\sigma \in\right.$ domain $h_{i-1}: \sigma \supset \rho$ and $h_{i-1}(\sigma)$ $\left.=h_{i}(\rho)\right\}$ is stationary. Set $S^{\prime}=\left\{\sigma \in S\right.$ : for all $\left.i<n, h_{i}(\sigma \mid n-i)=h_{n}(\varnothing)\right\}$.

(c) Define $\sigma_{\alpha}(i)$ by induction on $(i, \alpha)$ with the lexicographic order.

For each $\delta \in E$, set $Y_{\delta}=\{f \in F: f(0)=\delta\}$. Since $\left\{Y_{\delta}: \delta \in E\right\}$ is a discrete family of closed sets, if $X$ were metrizable, there would be a disjoint family $\left\{U_{\delta}: \delta \in E\right\}$ of open sets with $Y_{\delta} \subset U_{\delta}$. Aiming for a contradiction, assume that $\left\{U_{\delta}: \delta \in E\right\}$ is such a family.

Let $T=\left\{\sigma \in \Sigma: B(\sigma) \subset U_{\sigma(0)}\right\}$. By Lemma 3(a), for some $n \in \omega, T \cap \Sigma_{n}$ contains a stafull set, $S$. Apply Lemma 3(b) $n$ times to get a stafull $S^{\prime} \subset S$ such that for all $\sigma, \sigma^{\prime} \in S^{\prime}$ and $i<n, \sigma(0)_{i}=\sigma^{\prime}(0)_{i}$. Next, apply Lemma 3(c) with $\beta=\kappa$ to obtain $W=\left\{\sigma_{\alpha}: \alpha<\kappa\right\}$. For $\sigma \in W$, enumerate $A(\sigma, n)$ as $\left\{Z(\sigma, \delta): \delta<\kappa_{n}\right\}$. 
Let us take $\rho, \tau \in W$ (with $\rho(0)<\tau(0)$ ) and investigate whether there is a function $g$ from $\mathscr{Z}\left(\rho^{*}\right)$ to $\{0,1\}$ such that $\langle g, \rho, \tau\rangle \in B(\rho) \cap B(\tau)$. Conditions (7), (8), (9), (10), and (11) are satisfied, so the assumption that $\left\{U_{\alpha}: \alpha \in E\right\}$ is disjoint means that (12) fails. Thus, for each pair $\rho, \tau \in W$, we have a pair $\delta, \eta$ and $n \in \omega$ such that

$$
\begin{gathered}
Z(\rho, \delta)=Z(\tau, \eta) \cap \Sigma^{\rho \mid m}, \\
\text { either } \rho \mid m \in Z(\rho, \delta) \text { and } \tau \mid m \notin Z(\tau, \eta), \\
\text { or } \quad \rho \mid m \notin Z(\rho, \delta) \text { and } \tau \mid m \in Z(\tau, \eta) .
\end{gathered}
$$

Since $\kappa>\left(2^{\kappa_{n}}\right)^{+}$, we can apply the Erdös-Rado theorem, $\kappa \rightarrow(3)_{\kappa_{n}}^{2}$ to get $\rho, \sigma, \tau \in W$ (with $\rho(0)<\sigma(0)<\tau(0))$ and $\delta, \eta<\kappa_{n}$, for which each pair $(\rho, \sigma),(\rho, \tau)$, and $(\sigma, \tau)$ satisfies (17) and the same alternative of (18). (In the case $\kappa=\omega$, we use Ramsey's theorem $\omega \rightarrow(\omega)_{n}^{m}$.) It follows from (17) that

$$
Z(\sigma, \eta) \cap \Sigma^{\rho \mid m}=Z(\rho, \delta) \cap \Sigma^{\rho \mid m}=Z(\tau, \eta) \cap \Sigma^{\rho \mid m}=Z(\sigma, \delta) \cap \Sigma^{\rho \mid m} .
$$

Now applying (18) yields $\sigma \mid m \in Z(\sigma, \eta)$ and $\sigma \mid m \notin Z(\sigma, \eta)$. This contradiction establishes that $\left\{U_{\delta}: \delta \in E\right\}$ is not disjoint, and hence that $X$ is not metrizable.

8. Normal, not collectionwise normal spaces. The problem of constructing a normal, nonmetrizable Moore space is a special case of the problem of constructing a normal, not collectionwise normal space. Bing solved the problem in this generality when he introduced the notion of collectionwise normal [B,Example G]. This example was modified to be perfect [B, Example H]; and metacompact [M]. These spaces are far from being Moore spaces; their characters are at least $2^{\kappa_{1}}$. (The character of a space is the least infinite cardinal, $\kappa$, such that every point has a neighborhood base of cardinality at most $\kappa$. First countable is synonymous with character $\omega$.)

The above construction apparently was considered very special until the mid 70's. A general construction was suggested to the author by Przymusiński's explanation [Pr] of the space in $\left[F_{2}\right]$. We start with a space, $F$, which is normal and has an uncountable discrete subset, $Y$. The simplest example is to let $F$ be an uncountable discrete space. Let $G$ be the set of functions from $\mathcal{T}$, the family of open subsets of $F$, to $\{0,1\}$. The point set of the new space, $X$, is $F \cup G$. Points of $G$ are isolated. Basic open neighborhoods of points of $F$ have the form $B(U, a)$, where $U \in \mathcal{T}$ and $a$ is a finite subset of $\mathcal{T} . B(U, a)=\{x \in F: x \in U\} \cup\{g \in G$ : for all $V \in a$, if $U \subset V$, then $g(V)=1$ and if $U \cup V=\varnothing$, then $g(V)=0\}$.

Towards showing that $X$ is normal, let $H$ and $K$ be disjoint closed subsets of $X$. First we note that it suffices to consider the case where $H$ and $K$ are disjoint closed subsets of $F$. Because $F$ is normal, there are disjoint open sets, $U, V$ where $H \subset U$ and $K \subset V$. Then $B(U,\{U\})$ and $B(V,\{U\})$ are disjoint open subsets of $X$ containing $H$ and $K$, respectively.

We next use the uncountable discrete subset, $Y$, to show that $X$ is not collectionwise normal. $\{\{y\}: y \in Y\}$ is a discrete collection of closed subsets of $X$; assume $\{y\} \subset B\left(U_{y}, a_{y}\right)$. Now $B\left(U_{y}, a_{y}\right)$ "has positive measure", hence the uncountable collection is not disjoint. Of course, we can replace the informal measure argument with a combinatorial argument using Ramsey's Theorem, $\omega \rightarrow(\omega)_{n}^{m}$, or with the $\Delta$-system lemma. 
The space defined above is not $T_{1}$. There are several solutions to this problem. Perhaps the simplest is to identify each $x \in F$ with the function $g_{x} \in G$, defined by $g(V)=1$ iff $x \in V$. When $F$ is an uncountable discrete space, this gives Bing's Example $\mathrm{G}$. Another way is to replace $G$ with a sequence of copies of $G$; basic open sets may exclude finitely many of these copies. Formally, $X=F \cup(\omega \times G)$; points of $X-F$ are isolated; and for $x \in F$ a basic open set is $B(U, a, n)=(\omega-n) \times$ $B(U, a)$. When $F$ is an uncountable discrete space, this gives Bing's Example $\mathrm{H}$.

Recall that our goal is to reduce the character. What is the character of the above spaces? Basic open sets have the form $B(U, a)$ where $a$ is a finite subset of $\sigma$. Because of the uncountable discrete set $Y$, the cardinality of $\mathcal{T}$, and hence the character of $X$, is at least $2^{\aleph_{1}}$.

The first successful attempt to reduce the character was George $\left[\mathbf{F}_{2}\right]$. From the point of view of this section, $F=\left\{(\alpha, \beta) \in D\left(\omega_{1}\right) \times \omega_{1}: \alpha<\beta\right\}$ where $D\left(\omega_{1}\right)$ is $\omega_{1}$ with the discrete topology and the second factor is $\omega_{1}$ with the usual, order, topology. We replace $G$ by a sequence $\left(G_{\gamma}\right)_{\gamma<\omega_{1}}$ of approximations to $G$. Let $F_{\gamma}=\{(\alpha, \beta) \in F: \beta<\alpha\}$; let $G_{\gamma}$ be the family of functions from the subspace topology $\mathcal{T}_{\gamma}$, on $F_{\gamma}$ to $\{0,1\}$. The point set of the space is $F \cup \cup\left\{G_{\gamma}: \gamma \in \omega_{1}\right\}$. Points of $\cup\left\{G_{\gamma}: \gamma \in \omega_{1}\right\}$ are isolated. A basic open neighborhood of $(\alpha, \beta) \in F$ has the form $B(U, a, \beta)$, where $U \in \mathcal{T}_{\beta}$ and $a$ is a finite subset of $\mathcal{T}_{\beta} . B(U, a, \beta)=\{g \in$ $G_{\gamma}: \gamma<\beta$ and for all $V \in A$, if $U \subset V$ then $g\left(V \cap F_{\gamma}\right)=1$ and if $V \cap U=\varnothing$, then $\left.g\left(V \cap F_{\gamma}\right)=0\right\}$. The proofs of normality and not collectionwise normality are not so trivial as in the first example, but still are straightforward. The point, of course, is the reduced character. Each $F_{\beta}$ is countable, hence $\left|\mathcal{F}_{\beta}\right|=2^{\aleph_{0}}$, and finally the character of the space is $2^{\aleph_{0}}$.

The next important development is the space of Navy [Na], which crossed the space, $M$, of $\left[F_{3}\right]$ with Bing's Example $G$. The space $M$ is essentially the space described in "Visualizing the space", with points $(\rho, \tau)$ retained rather than replaced by cubes. $M$ is not normal; Navy's idea is to replace the point $(\rho, \tau)$ by a copy of Bing's Example $G$ indexed by $(\rho, \tau)$. Now, normality is built-in (see below); paraLindelöfness follows quickly.

Another view of Navy's space is the one emphasized in this section. Let $F$ be the set of functions from $\omega$ to $\omega_{1}$. In the product topology, $F$ is a nonseparable metrizable space, a fortiori, $F$ is normal and has an uncountable discrete subspace. We replace $G$ by copies of $G$ indexed by entwined pairs. Formally $X=F \cup$ $\{(g, \rho, \tau): g \in G$ and for some $n \in \omega, \rho(0)<\tau(0)<\rho(1)<\cdots<\rho(n-1)<$ $\tau(n-1)\}$. Points of the form $(g, \rho, \tau)$ are isolated. Basic open neighborhoods of points $x \in F$ have the form $B(\sigma, a)$ where $[\sigma]$ is a basic open subset of $F$, and $a$ is a finite set of arbitrary open subsets of $F$.

To demonstrate normality, it suffices to consider the case where the disjoint closed sets, $H, K$, are subsets of $F$. Since $F$ is normal there are disjoint open subsets $U, V$, of $F$ satisfying $H \subset U$ and $K \subset V$. Then $\cup\{B(\sigma,\{U\}):[\sigma] \subset U\}$ and $\cup\{B(\sigma,\{U\}):[\sigma] \subset V\}$ are disjoint open sets separating $H$ and $K$ in $X$. That $X$ is not collectionwise normal is as in $\$ 7$; we can use the simpler notion full rather than stafull (replace stationary with uncountable in the definition of stafull). 
As defined above, the space has character $2^{\aleph_{1}}$. Reducing the character to $2^{\aleph_{0}}$ is easy; we restrict a parameter $a$ to range over finite subsets of countable unions of basic open subsets of $F$. In the proof of normality, we cannot use $U$ in $a$, but for each $\sigma$, there is a "countable approximation" to $U$ (depending on $\sigma$ ) which works because of the entwining.

The penultimate step in the development of the space presented in this paper was to assume $\mathrm{CH}$ and enumerate the countable unions of basic open subsets of $F$. The final step, of course, was to replace $\mathrm{CH}$ with HYP.

\section{BIBLIOGRAPHY}

[B] R. H. Bing, Metrization of topological spaces, Canad. J. Math. 3 (1951), 175-186.

[DJ] K. Devlin and R. Jensen, Marginalia to a theorem of Silver, ISLIC Logic Conf., Lecture Notes in Math., vol. 499, Springer-Verlag, Berlin and New York, 1975, pp. 115-142.

[JD] A. Dodd and R. Jensen, The core model, Ann. Math. Logic 20 (1981), 43-75.

[E] R. Engelking, General topology, Polish Scientific Publishers, Warsaw, 1977.

[ $\left.\mathbf{F}_{1}\right]$ W. G. Fleissner, Normal Moore spaces in the constructible universe, Proc. Amer. Math. Soc. 46 (1974), 294-298.

$\left[\mathbf{F}_{2}\right] \ldots$, A normal, collectionwise Hausdorff, not collectionwise normal space, Topology Appl. 6 (1976), 57-64.

$\left[\mathbf{F}_{3}\right] \_$A collectionwise Hausdorff, non normal Moore space with a o-locally countable base, Topology Proc. 4 (1979), 83-96.

[F $\left.\mathbf{F}_{4}\right]$ W. G. Fleissner, Normal Moore spaces and large cardinals, Handbook of Set-Theoretic Topology,

[Je] T. Jech, Set theory, Academic Press, New York, 1977.

(Kunen and Vaughan, Eds.), North-Holland, Amsterdam (to appear).

[J] F. B. Jones, Concerning normal and completely normal spaces, Bull. Amer. Math. Soc. 47 (1937), $671-677$.

[K] K. Kunen, Set theory: An introduction to independence proofs, North-Holland, New York, 1980.

[M] E. Michael, Point-finite and locally-finite covers, Canad. J. Math. 7 (1955), 275-279.

[Mt] W. Mitchell, Hypermeasurable cardinals, Studies in Logic 97 (1979), 303-316.

[Na] C. Navy, ParaLindelöf versus paracompact, Topology Appl. (to appear).

[N] P. J. Nyikos, A provisional solution to the normal Moore space problem, Proc. Amer. Math. Soc. 78 (1980), 429-435.

[Pr] T. C. Przymusinski, Collectionwise Hausdorff property in product spaces, Colloq. Math. 36 (1976), 49-56.

$\left[\mathbf{T}_{1}\right]$ F. D. Tall, Set theoretic consistency results and topological theorems, Dissertationes Math. 148 (1977), 1-53.

$\left[\mathbf{T}_{2}\right]$ _ The normal Moore space problem, Math. Centre Tracts 116 (1979), 243-261.

Department of Mathematics and Statistics, University of Pittsburgh, Pittsburgh, PennsylVANIA 15260 